TITLE:

\title{
Manipulation of resonant Auger processes using a strong bichromatic field
}

\section{AUTHOR(S):}

Chatterjee, Souvik; Nakajima, Takashi

\section{CITATION:}

Chatterjee, Souvik ...[et al]. Manipulation of resonant Auger processes using a strong bichromatic field. Physical Review A 2015, 91(4): 043413.

ISSUE DATE:

2015-04-21

URL:

http://hdl.handle.net/2433/198436

RIGHT:

(C2015 American Physical Society 
PHYSICAL REVIEW A 91, 043413 (2015)

\title{
Manipulation of resonant Auger processes using a strong bichromatic field
}

\author{
Souvik Chatterjee and Takashi Nakajima* \\ Institute of Advanced Energy, Kyoto University, Gokasho, Uji, Kyoto 611-0011, Japan
}

(Received 4 March 2015; published 21 April 2015)

\begin{abstract}
We theoretically investigate the effects of strong couplings in resonant Auger processes under the combination of strong resonant x-ray and nearly resonant optical pulses. The x-ray field couples the ground state with a core-excited state, while the optical field couples the core-excited state with another core-excited state of opposite parity. The Auger electron spectrum changes its shape as the intensities of the x-ray and/or optical fields increase, and at sufficiently high intensities we observe that the splitting, which is induced by the optical field, is superposed on the asymmetric splitting induced by the $\mathrm{x}$-ray field in the Auger electron spectra. The asymmetric splitting itself, which is induced by the strong $\mathrm{x}$-ray pulse, is persistent but modified due to the presence of the strong optical field. Moreover, through the systematic study by including or excluding the individual photoionization processes from the core-excited states and the direct photoionizaton process from the ground state, we clarify the contribution of the respective processes to the total electron yield and the Auger electron spectra. These results show that we can manipulate the resonant Auger processes through the introduction of the second core-excited state and the strong optical field.
\end{abstract}

DOI: 10.1103/PhysRevA.91.043413

\section{INTRODUCTION}

The study of the dynamics of core-excited states induced by short-wavelength radiation has been attracting a lot of interest in recent years. Since the core-electron dynamics usually occur in an ultrafast time scale and synchrotron radiation was the main light source to excite the core electrons, time-resolved detection of such processes was a difficult task for many years. However, the advent of $\mathrm{x}$-ray free-electron lasers (XFELs) enables us to undertake a thorough study from both an experimental and a theoretical point of view.

The exposure of an atom to $x$-ray radiation at the appropriate wavelength resonantly creates a core-excited state with a lifetime of a few femtoseconds. Upon creation of the coreexcited state, the outer valence electron immediately fills up the vacancy in the inner shell, and the excess energy coming out of this process results in the electron emission from the outer valence shell (Auger decay). This Auger process is specifically called spectator Auger decay, because the electron excited from the core to the outmost shell is not involved in the Auger decay [1]. Another type of Auger process is called participator Auger decay, in which the electron excited from the core to the outmost shell also participates in the Auger decay [1]. However, the participator Auger decay is generally much weaker than the spectator Auger decay. In particular, the Auger (spectator/participator) process that follows the resonant excitation to the core-excited state is called a resonant Auger (RA) process [1]. In addition to the RA process, an exposure to $\mathrm{x}$-ray radiation also induces direct nonresonant photoionization by promoting one electron into the continuum and other electron into an excited state. The excited ionic state formed this way is a common final state for both the direct photoionization and spectator Auger processes. This means that the two processes, the core-excitation followed by the RA process and the direct nonresonant photoionization process, can interfere with each other, in principle. However,

\footnotetext{
*nakajima@iae.kyoto-u.ac.jp
}

PACS number(s): $33.20 . \mathrm{Xx}, 32.80 . \mathrm{Hd}$, 41.60.Cr, 82.50.Kx

the interference is usually extremely weak, since two electrons must be involved for the direct nonresonant photoionization to occur and accordingly, the probability of such a process is usually much smaller than that of the core excitation followed by the RA process. Nevertheless, a signature of interference between these two processes in the Auger electron spectra has been reported in earlier studies [2-5]. The evidence of such interference has been more clearly observed in the angleresolved Auger electron spectra [6,7]. The use of an intense $\mathrm{x}$-ray field has opened an opportunity to study new aspects of RA processes [8-11]. In particular, such an intense x-ray field can induce more significant interference [9-12], because the probability of the RA process does not further increase once the Rabi oscillation sets in under a sufficiently intense $\mathrm{x}$-ray field $[8,11]$, while that of the direct photoionization process continuously increases until complete ionization takes place.

In addition to the studies related to RA decay [2-13] under the single-color field as mentioned above, several investigations have been performed to study the RA process under the two-color (x-ray + optical) field $[14,15]$. For the two-color scheme there are a few different ways to utilize the optical field. One way is to induce two-photon ionization from the core-excited state to control the product ratio between the singly and doubly charged final ionic states through resonant and normal Auger decays [16]. Another way is to resonantly couple the two core-excited states with opposite parity so that the population transfer is induced between them, resulting in the significant change in the RA spectra [17]. The other way is to strongly couple two core-excited states with opposite parity to induce electromagnetically induced transparency in the $\mathrm{x}$-ray wavelength region $[18,19]$. We should also mention that, irrespective of the primary objective to employ the two-color scheme for the RA process, an introduction of the strong optical field results in the formation of sidebands around the Auger peak in the RA spectra $[14,15,17,20-25]$. This is known as laser-assisted Auger decay.

The purpose of this work is to theoretically investigate the dynamics of the RA process involving the two core-excited states with opposite parity under the strong bichromatic 
(x-ray + optical) field. More specifically, we investigate in what way the strong optical coupling between the two coreexcited states under the simultaneous presence of the strong $\mathrm{X}$-ray pulse influences the Auger electron spectra. In addition to the strong coupling effects, the x-ray and optical pulses also induce significant photoionization from the core-excited states. Through the systematic calculations by including or excluding the individual photoionization processes from the core-excited states, we clarify the contribution of the respective photoionization channels on the Auger electron spectra. Related to our work we note that a similar process has been studied in Ref. [17] with a bichromatic field to demonstrate the efficient manipulation of population in the core-excited state. However, the direct nonresonant photoionization process, which is essential to see the interference effect [2-7], is not included in their model, and moreover, the employed x-ray pulse is weak. In contrast, our model incorporates the direct nonresonant photoionization process and the employed x-ray pulse is intense enough to induce a strong interference effect [9-12], which can be confirmed by the appearance of an asymmetric profile in the Auger electron spectra [11].

\section{THEORY}

In order to theoretically describe the RA processes under the bichromatic field, we solve the time-dependent Schrödinger equation for the neon atom. Figure 1 depicts the relevant atomic energy levels along with the associated radiative and nonradiative couplings. This model contains the ground and two core-excited states. The ground state, $|g\rangle\left(1 s^{2} 2 s^{2} 2 p^{6}\right.$ at

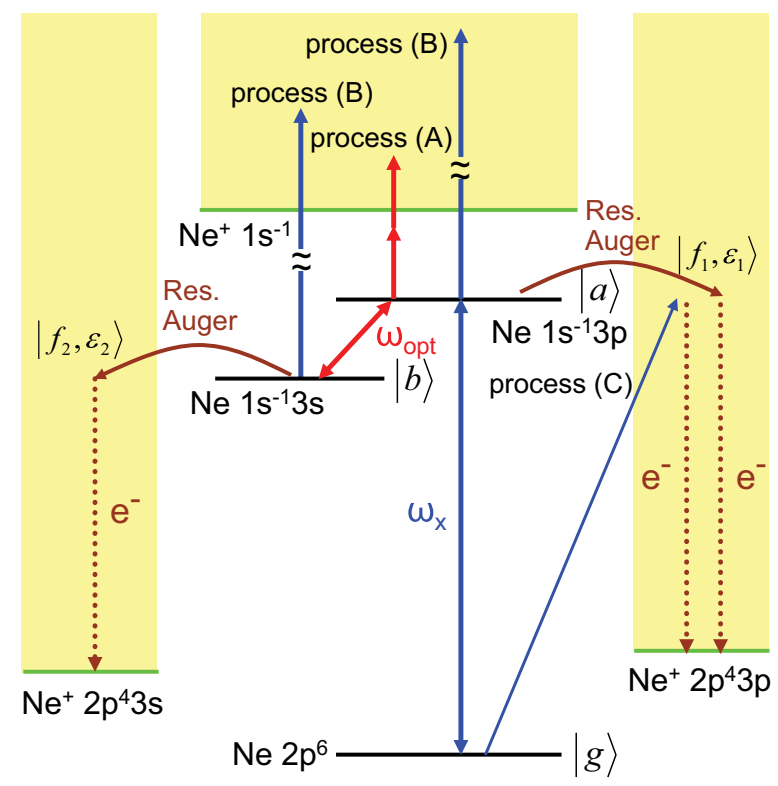

FIG. 1. (Color online) Level scheme under consideration. A resonant X-ray pulse couples the ground state $|g\rangle$ and a core-excited state $|a\rangle$, and an optical pulse couples the core-excited state $|a\rangle$ and another core-excited state with opposite parity $|b\rangle$. Auger decay takes place from the core-excited states $|a\rangle$ and $|b\rangle$ into the continuum states $\left|f_{1}, \varepsilon_{1}\right\rangle$ and $\left|f_{2}, \varepsilon_{2}\right\rangle$, respectively. A direct ionization pathway, which plays a very important role in this work, is also explicitly shown. Processes (A)-(C) describe photoionization, as explained in the text.
$0 \mathrm{eV})$, is resonantly coupled to the core-excited state, $|a\rangle$ $\left(1 s^{-1} 3 p\right.$ at $867.1 \mathrm{eV}$ ), by the $\mathrm{x}$-ray pulse (photon energy 867.1 $\mathrm{eV})$. The core-excited state, $|a\rangle$, is further coupled to another core-excited state with opposite parity, $|b\rangle\left(1 s^{-1} 3 s\right.$ at 865.2 $\mathrm{eV}$ ), by the optical pulse (photon energy $1.55 \mathrm{eV}$ ). The two core-excited states, $|a\rangle$ and $|b\rangle$, decay into the continuum states $\left|f_{1}, \varepsilon_{1}\right\rangle\left(\mathrm{Ne}^{+} 2 p^{4} 3 p\right.$ at $55.8 \mathrm{eV}+$ free electron $)$ and $\left|f_{2}, \varepsilon_{2}\right\rangle$ $\left(\mathrm{Ne}^{+} 2 p^{4} 3 s\right.$ at $52.1 \mathrm{eV}+$ free electron), respectively, through the RA decay. Here, $\varepsilon_{k}(k=1,2)$ is the energy of the free electron emitted into the continuum, and $f_{k}(k=1,2)$ stands for the states of ion, $\mathrm{Ne}^{+}$, left after the Auger decay from states $|a\rangle$ and $b\rangle$, respectively.

Processes (A) and (B) described in Fig. 1 are the photoionization processes into the continuum $\left(\mathrm{Ne}^{+} 1 s^{-1}\right.$ at $870.1 \mathrm{eV}+$ free electron) from $|a\rangle$ by the optical pulse and from $|a\rangle$ and $|b\rangle$ by the x-ray pulse, respectively. The third process $(\mathrm{C})$ depicted in Fig. 1 is the direct photoionization into the continuum $\left(\mathrm{Ne}^{+} 2 p^{4} 3 p\right.$ at $55.8 \mathrm{eV}+$ free electron) from $|g\rangle$ by the X-ray pulse. We often refer to these processes in Sec. III.

The time-dependent wave function $\Psi(t)$ of the system shown in Fig. 1 can be written as

$$
\begin{aligned}
\Psi(t)= & c_{g}(t)|g\rangle+e^{-i \omega_{x} t} c_{a}(t)|a\rangle+e^{-i\left(\omega_{x}-\omega_{\mathrm{opt}}\right) t} c_{b}(t)|b\rangle \\
& +\int e^{-i \omega_{x} t} c_{f_{1}}\left(\varepsilon_{1}, t\right)\left|f_{1}, \varepsilon_{1}\right\rangle d \varepsilon_{1} \\
& +\int e^{-i\left(\omega_{x}-\omega_{\mathrm{opt}}\right) t} c_{f_{2}}\left(\varepsilon_{2}, t\right)\left|f_{2}, \varepsilon_{2}\right\rangle d \varepsilon_{2},
\end{aligned}
$$

where $c_{j}(t)(j=g, a, b)$ and $c_{f_{k}}(t)(k=1,2)$ are the timedependent amplitudes of the states indicated by the subscripts. The central frequencies of the x-ray and optical laser pulses are represented by $\omega_{x}$ and $\omega_{\text {opt }}$, respectively. The time-dependent dynamics of the system under the bichromatic field is described by the following set of coupled differential equations obtained under the rotating-wave approximation. Since we are not concerned with the Auger electron profile associated with state $|b\rangle$, we have omitted the equation for the state $\left|f_{2}, \varepsilon_{2}\right\rangle$. The equations read

$$
\begin{aligned}
i \dot{c}_{g}(t)= & {\left[E_{g}-\frac{i}{2} \gamma_{g}(t)\right] c_{g}(t) } \\
& +\left[D_{a g}^{\dagger}-i \pi D_{f_{1} g}^{\dagger}(t) V_{f_{1} a}\right] c_{a}(t) \\
i \dot{c}_{a}(t)= & {\left[E_{a}+S_{a}-\omega_{x}-\frac{i}{2}\left(\Gamma_{a}+\gamma_{a}(t)\right)\right] c_{a}(t) } \\
& +\left[D_{a g}-i \pi V_{f_{1} a}^{\dagger} D_{f_{1} g}(t)\right] c_{g}(t) \\
& +D_{b a}^{\dagger} c_{b}(t) \\
i \dot{c}_{b}(t)=\left[\begin{array}{c}
E_{b}+ \\
+
\end{array}\right. & \left.S_{b}-\left(\omega_{x}-\omega_{\mathrm{opt}}\right)-\frac{i}{2}\left(\Gamma_{b}+\gamma_{b}(t)\right)\right] c_{b}(t) \\
+ & D_{b a} c_{a}(t) \\
i \dot{c}_{f_{1}}\left(\varepsilon_{1}, t\right)= & {\left[E_{f_{1}}+\varepsilon_{1}-\omega_{x}\right] c_{f_{1}}\left(\varepsilon_{1}, t\right)+D_{f_{1} g}(t) c_{g}(t) } \\
& +V_{f_{1} a} c_{a}(t)
\end{aligned}
$$

which is an extended version of the equations derived for the system consisting of the ground and single core-excited 
states coupled by the x-ray pulse [11]. In the above equations $E_{j}$ are the energies of states $|j\rangle(j=g, a, b)$, and $E_{f_{1}}$ is the energy of the final ionic state after the Auger decay. $\Gamma_{k}$ are the total Auger decay rates of the states $|k\rangle(k=a, b)$. $\gamma_{g}(t)$ is the direct photoionization width from the ground state by the x-ray field, while $\gamma_{k}(t)$ are the photoionizaton widths from the core-excited states $|k\rangle(k=a, b)$ by the bichromatic field. The stark shifts of state $|k\rangle(k=a, b)$ induced by the optical field are given by $S_{k}$. The Rabi frequencies, $D_{j k}$ ( $j=a, b$ and $k=g, a)$, between states $|j\rangle$ and $|k\rangle$ are defined by

$$
\begin{gathered}
D_{a g}=\frac{1}{2} \mu_{a g} E_{x}(t), \\
D_{b a}=\frac{1}{2} \mu_{b a} E_{\mathrm{opt}}(t),
\end{gathered}
$$

in which $\mu_{j k}(j=a, b$ and $k=g, a)$ are the transition dipole matrix elements between state $|j\rangle$ and $|k\rangle$, and $E_{x}(t)$ and $E_{\text {opt }}(t)$ are the time-dependent amplitudes of the x-ray and optical fields, respectively. In this paper we assume that both laser pulses have Gaussian temporal profiles, i.e.,

$$
\begin{gathered}
E_{x}(t)=E_{0, x} \exp \left[-4 \ln 2\left(\frac{t}{\tau_{x}}\right)^{2}\right], \\
E_{\text {opt }}(t)=E_{0, \text { opt }} \exp \left[-4 \ln 2\left(\frac{t}{\tau_{\text {opt }}}\right)^{2}\right],
\end{gathered}
$$

where $\tau_{x}\left(\tau_{\mathrm{opt}}\right)$ is a duration of the X-ray (optical) pulse defined by the FWHM for the envelope of the field amplitude. $E_{0, x}$ and $E_{0, \text { opt }}$ are the peak amplitudes of the x-ray and optical fields, respectively. The transition moment from states $|g\rangle$ to $\left|f_{1}, \varepsilon_{1}\right\rangle$ induced by the $\mathrm{x}$-ray pulse as a result of the direct ionization into the continuum defined by $\left|f_{1}, \varepsilon_{1}\right\rangle$ can be expressed as

$$
D_{f_{1} g}\left(\varepsilon_{1}, t\right)=\frac{1}{2} \mu_{f_{1} g}\left(\varepsilon_{1}\right) E_{x}(t),
$$

where $\mu_{f_{1} g}$ is the transition matrix element between the continuum states $\left|f_{1}, \varepsilon_{1}\right\rangle$ and the ground state $|g\rangle$.

Finally, the $V_{f_{1} a}$ and $V_{f_{2} b}$ terms which appear in Eqs. (2)-(5) represent the Coulomb matrix elements between the core-excited states, $|a\rangle$ and $|b\rangle$, and the associated continua, $\left|f_{1}, \varepsilon_{1}\right\rangle$ and $\left|f_{2}, \varepsilon_{2}\right\rangle$, respectively, as a result of the Auger decay. According to the Wigner-Weisskopf theory [26,27], $V_{f_{1} a}$ and $V_{f_{2} b}$ can be connected to the partial Auger decay rates, $\Gamma_{f_{1}}^{(a)}$ and $\Gamma_{f_{2}}^{(b)}$, i.e.,

$$
\begin{aligned}
& \Gamma_{f_{1}}^{(a)}\left(\varepsilon_{1}\right)=2 \pi\left|V_{f_{1} a}\left(\varepsilon_{1}\right)\right|^{2}, \\
& \Gamma_{f_{2}}^{(b)}\left(\varepsilon_{2}\right)=2 \pi\left|V_{f_{2} b}\left(\varepsilon_{2}\right)\right|^{2} .
\end{aligned}
$$

The Coulomb matrix elements, $V_{f_{1} a}$ and $V_{f_{2} b}$, vary slowly with $\varepsilon_{k}(k=1,2)$ [1] because of the high kinetic energy of the Auger electrons. As a result, we can safely assume that $V_{f_{1} a}$ and $V_{f_{2} b}$ are constant over the continuum energies, $\varepsilon_{1}$ and $\varepsilon_{2}$, respectively.

In deriving Eqs. (2)-(5), we have neglected, for simplicity, the continuum-continuum couplings induced by the optical pulse. Such couplings modify the dynamics of the free electrons emitted through the Auger processes [14,15,17,20-25]. However, the modifications in the Auger electron spectrum primarily appear as sidebands around the main Auger peak. In this work, we neglect the continuum-continuum couplings, since the main objective of our work is to investigate the modification of the main Auger peak under the presence of the bichromatic field. The effects of the optical field on the final ionic states after the Auger decay are expected to be very small [17], and accordingly, we have not included it in our theoretical model.

After a sufficiently long time the population in the coreexcited states will completely decay into the continuum states, and hence we can calculate the total electron yield $P_{\text {tot }}$ from the following relation:

$$
P_{\text {tot }}=1-\lim _{t \rightarrow \infty}\left|c_{g}(t)\right|^{2} .
$$

The Auger electron spectra $P\left(\varepsilon_{1}\right)$ associated with state $|a\rangle$ is calculated from the relation of

$$
P\left(\varepsilon_{1}\right)=\lim _{t \rightarrow \infty}\left|c_{f_{1}}\left(\varepsilon_{1}, t\right)\right|^{2} .
$$

\section{NUMERICAL RESULTS AND DISCUSSIONS}

Prior to presenting the numerical results, we describe the assumptions and specify the parameters under which we perform the calculations. The photon energy and the durations of the X-ray and optical pulses are, respectively, $867.1 \mathrm{eV}$ (resonant with the $|g\rangle-|a\rangle$ transition) and $1.55 \mathrm{eV}$ (offresonant with the $|a\rangle-|b\rangle$ transition), and 2.4 fs (comparable to the lifetime of the core-excited states) and $20 \mathrm{fs}$, unless otherwise mentioned (see Fig. 2). The employed dipole matrix elements for the relevant states of $\mathrm{Ne}$ atom are $\mu_{a g}=0.0096$ (a.u.) and $\mu_{b a}=2.8$ (a.u.), respectively [17], and the lifetimes of the core-excited states are 2.41 fs for both $|a\rangle$ and $|b\rangle$. The partial widths of the two core-excited states are assumed to be constant over the continuum energies, $\varepsilon_{1}$ and $\varepsilon_{2}$, and $\Gamma_{f_{1}}^{(a)}=\Gamma_{f_{2}}^{(b)}=0.086 \mathrm{eV}$ [17]. The direct ionization width for the ground state $\gamma_{g}$ is calculated using the value reported in Ref. [11]. The ionization widths for the x-ray and optical pulses $\gamma_{a}$ and $\gamma_{b}$, respectively, and the Stark shift induced by the optical pulse for the core-excited states $S_{a}$ and $S_{b}$ are calculated using the values reported in Ref. [17]. The ionization width

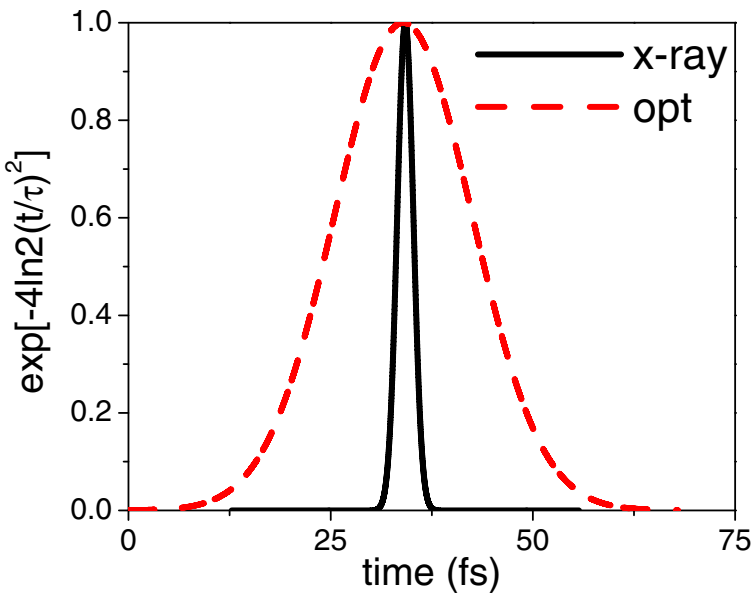

FIG. 2. (Color online) The temporal profiles of the x-ray (solid) and optical (dashed) pulses. They have Gaussian shapes with FWHM of 2.4 and $20 \mathrm{fs}$, respectively. 


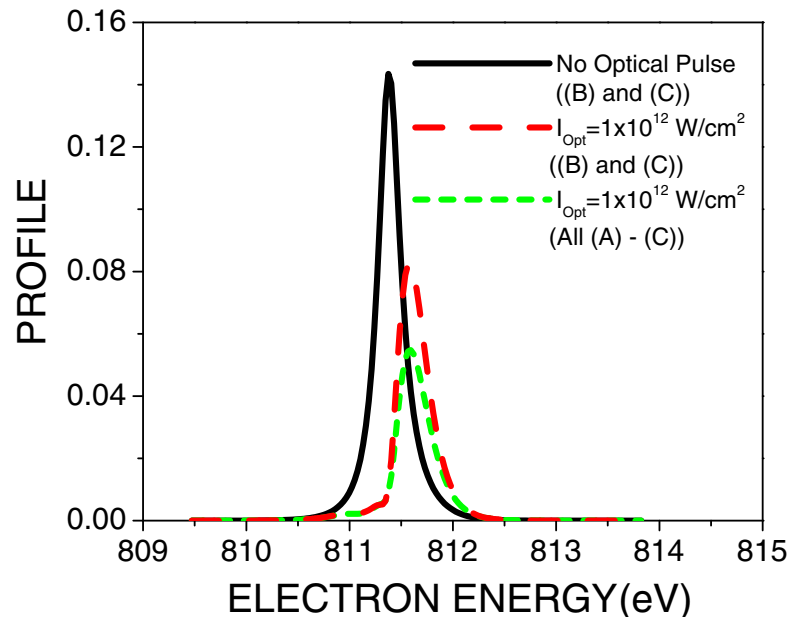

FIG. 3. (Color online) Auger electron spectra in the weak intensity regime of the x-ray pulse. The optical pulse intensities are $I_{\mathrm{opt}}=0$ [solid, with processes (B) and (C)], $I_{\mathrm{opt}}=10^{12} \mathrm{~W} / \mathrm{cm}^{2}$ [dashed, with processes (B) and (C)], and $I_{\mathrm{opt}}=10^{12} \mathrm{~W} / \mathrm{cm}^{2}$ [short-dashed, with all processes $(\mathrm{A})-(\mathrm{C})$ ], respectively.

and the Stark shift of state $|b\rangle$ are assumed to be zero. Note that the processes $(\mathrm{A})-(\mathrm{C})$ we sometimes refer to in the following discussions are the three photoionization processes shown in Fig. 1.

Using the parameters described above, we numerically solve Eqs. (2)-(5) by the fourth-order Runge-Kutta method to study the dynamics of the RA processes under the bichromatic field. We first consider the case in which the intensity of the $\mathrm{x}$-ray pulse is weak in a sense that a very small fraction of the ground-state population is excited by the $\mathrm{x}$-ray pulse. For the fixed $\mathrm{x}$-ray pulse intensity at $10^{15} \mathrm{~W} / \mathrm{cm}^{2}$, we perform the calculations without and with the optical pulse at $10^{12} \mathrm{~W} / \mathrm{cm}^{2}$. To clarify the influence of the ionization process from $|a\rangle$ by the optical pulse [process (A)], we also carry out an additional calculation by setting the ionization width from $|a\rangle$ by the optical pulse to be zero. The results are shown in Fig. 3. We notice that the introduction of the optical pulse results in not only the shift of the peak position of the Auger electrons (dashed and short-dashed lines in Fig. 3), but also the reduction of the peak height itself (short-dashed line in Fig. 3). This is because the optical pulse induces ionization [process (A)] from state $|a\rangle$, which results in the reduction of population in state $|a\rangle$ before the Auger decay takes place.

We now increase the intensity of the $\mathrm{x}$-ray pulse under the presence of an optical pulse at $10^{12} \mathrm{~W} / \mathrm{cm}^{2}$. As the intensity of the $\mathrm{x}$-ray pulse increases from $10^{18} \mathrm{~W} / \mathrm{cm}^{2}$ to $5 \times 10^{18} \mathrm{~W} / \mathrm{cm}^{2}$, the line splitting associated with the Rabi oscillations induced by the X-ray pulse between $|g\rangle$ and $|a\rangle$ $[8,11,12]$ gradually appear in the Auger electron spectra, as shown in Fig. 4. The origin of the multiple peak structure in Fig. 4 has been interpreted as dynamic interference of electron wave packets of the same kinetic energy emitted at different times during the $\mathrm{x}$-ray pulse [28]. The reason such high intensity for the x-ray pulse is necessary to observe the line splitting is that, obviously, the dipole moment between $|g\rangle$ and $|a\rangle$ is very small and the x-ray pulse duration we have assumed is also very short, $2.4 \mathrm{fs}$. By the careful examination of

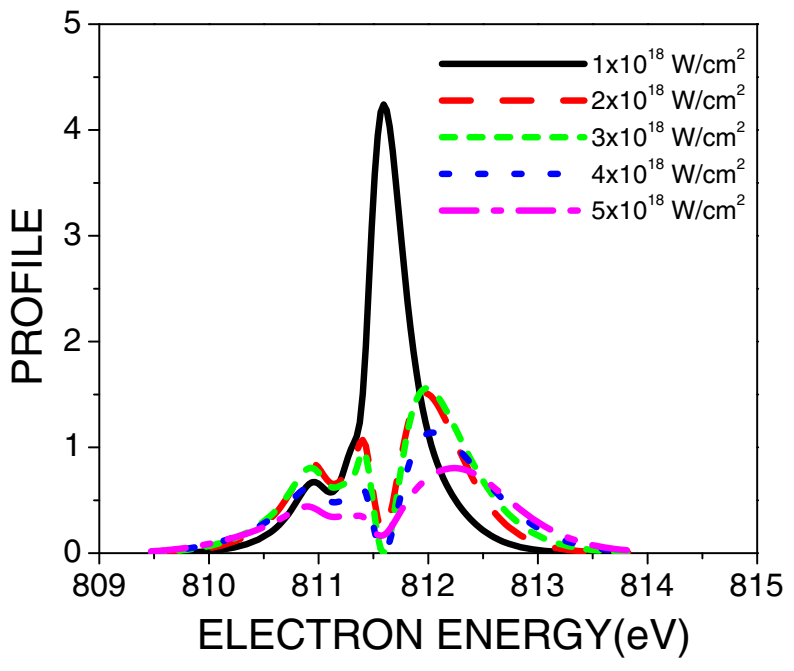

FIG. 4. (Color online) Auger electron spectra in the strong intensity regime of the $\mathrm{x}$-ray pulse. The optical pulse intensity is $I_{\mathrm{opt}}=10^{12} \mathrm{~W} / \mathrm{cm}^{2}$. The x-ray pulse intensities are $I_{x}=10^{18}$ (solid), $2 \times 10^{18}$ (dashed), $3 \times 10^{18}$ (short-dashed), $4 \times 10^{18}$ (dotted), and $5 \times 10^{18} \mathrm{~W} / \mathrm{cm}^{2}$ (dot-dashed), respectively.

Fig. 4 we also notice another line splitting in the Auger electron spectra at around $811.4 \mathrm{eV}$. This line splitting becomes more subtle as the $\mathrm{x}$-ray pulse intensity increases. Thus this line splitting becomes more visible only when the intensities of the x-ray and optical pulses are both appropriate, because the sufficiently high intensities of the x-ray and/or optical pulses result in rapid ionization from $|a\rangle$, which obscures the line splitting buried in the shoulder of the peak at $811.4 \mathrm{eV}$.

In our study of the RA processes with two core-excited states under the action of the bichromatic field, three different photoionization processes [processes (A)-(C) in Fig. 1] are also involved. But the respective contribution to the total electron yield defined by Eq. (13) is not very clear. To clarify their respective contributions we solve Eqs. (2)-(5) by including processes (A)-(C) step by step. The results are summarized in Fig. 5. We first look into the result for which laser-induced ionization processes (A)-(C) are all excluded [dot-dashed line in Fig. 5(a)]. Under this condition the total electron yield is equal to the total Auger electron yield via states $|a\rangle$ and $|b\rangle$. Interestingly, we notice that the total electron yield decreases as the optical pulse intensity increases. This is a kind of coherent population trapping (CPT) due to the simultaneous presence of the coherent $\mathrm{x}$-ray pulse and the optical pulse to couple $|g\rangle-|a\rangle$ and $|a\rangle-|b\rangle$, respectively. By comparing the temporal evolution of populations in states $|g\rangle$, $|a\rangle$, and $|b\rangle$ at the x-ray pulse intensity of $5 \times 10^{18} \mathrm{~W} / \mathrm{cm}^{2}$ with the optical pulse off [Fig. 5(b)] and on [Fig. 5(c)] with processes (A)-(C) all excluded in both figures, we can ensure that CPT indeed takes place if the optical pulse is sufficiently intense [Fig. 5(c)]. However, CPT in our case is imperfect, as we can clearly see in Fig. 5(c), and there is an appreciable population loss even at the optical pulse intensity of $6 \times 10^{12} \mathrm{~W} / \mathrm{cm}^{2}$ [dashed line in Fig. 5(c)]. There are two reasons for this. The first reason is that our system is very lossy due to the presence of the Auger processes. The second reason 

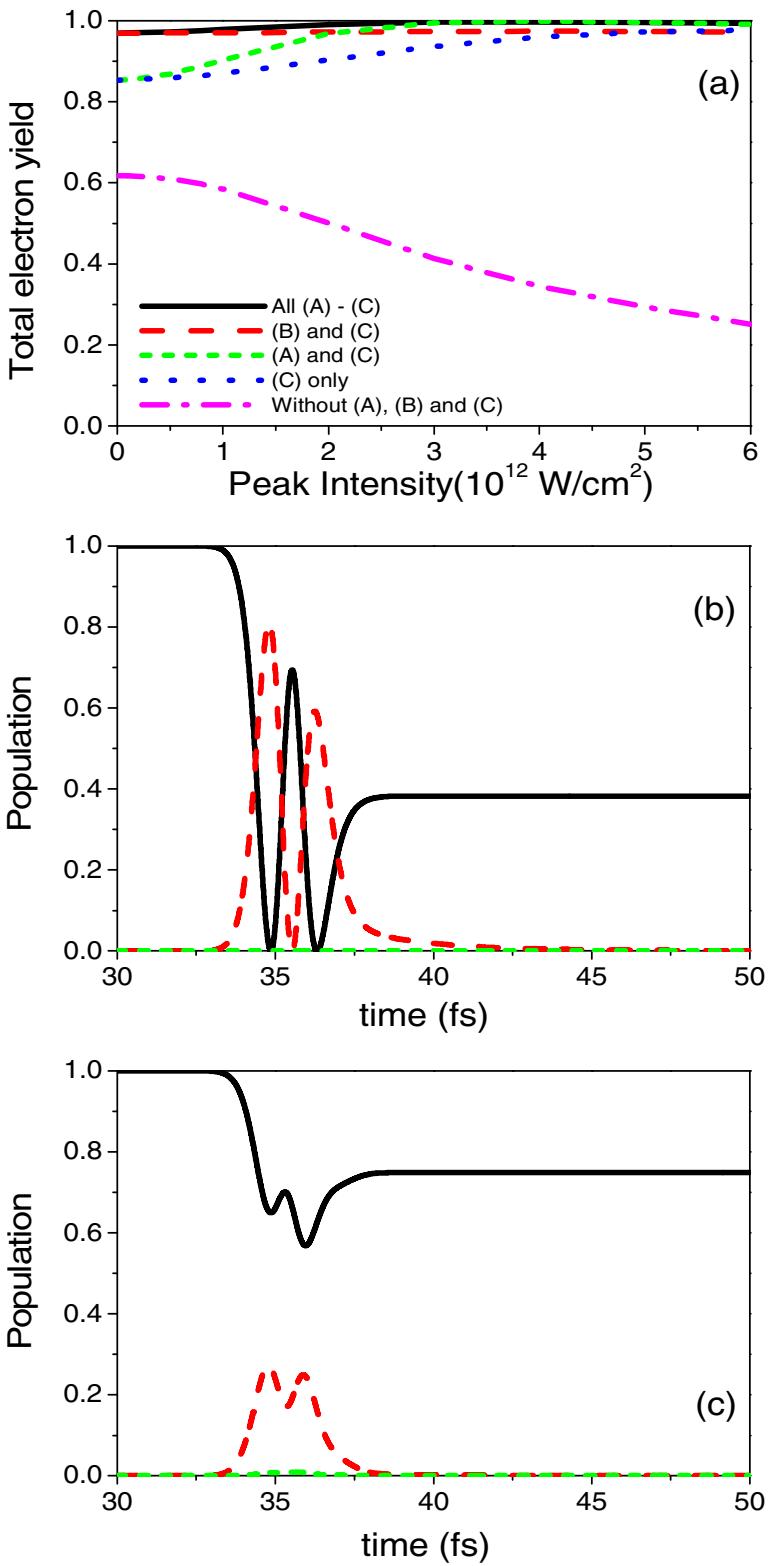

FIG. 5. (Color online) Total electron yield and the population dynamics for the system shown in Fig. 1 under different conditions. (a) Variation of the total electron yield $P_{\text {tot }}$ as a function of peak intensity of the optical pulse under different conditions. Results by taking into account all photoionization processes (A)-(C) (solid), processes (B) and (C) only (dashed), processes (A) and (C) only (short-dashed), process (C) only (dotted), and all processes (A)-(C) excluded (dot-dashed) are presented. (b) Temporal dynamics of the populations in states $|g\rangle$ (solid), $|a\rangle$ (dashed), and $|b\rangle$ (short-dashed) with $I_{x}=5 \times 10^{18} \mathrm{~W} / \mathrm{cm}^{2}$ and optical pulse off. (c) Similar to panel (b) but with optical pulse on at $I_{\mathrm{opt}}=6 \times 10^{12} \mathrm{~W} / \mathrm{cm}^{2}$. Laser-induced ionization processes (A)-(C) are all excluded in both (b) and (c).

is that the optical pulse is not at resonance with the $|a\rangle-|b\rangle$ transition.

We now include the ionization process from state $|g\rangle$ directly into the continuum [process $(\mathrm{C})$ ] by the X-ray pulse but still exclude the laser-induced ionization from the excited states [processes (A) and (B)]. In this case CPT becomes more imperfect due to the loss of population from $|g\rangle$ directly

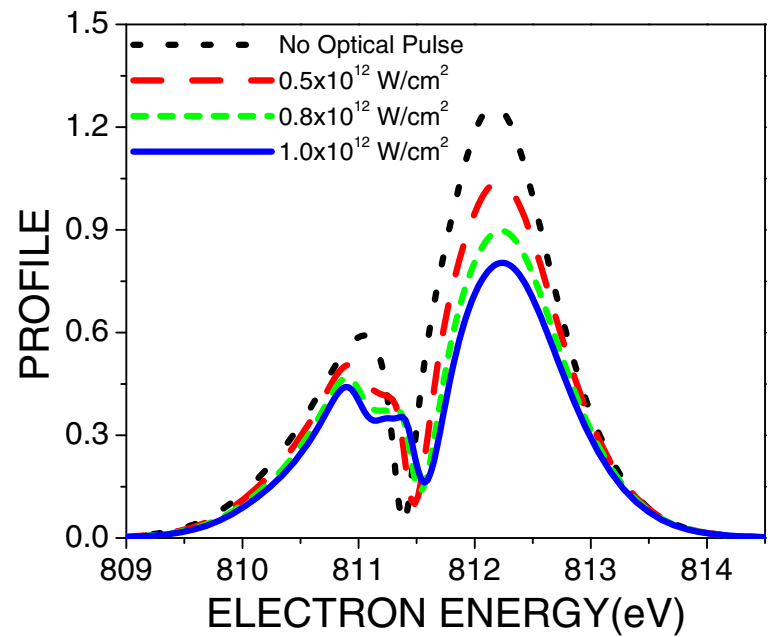

FIG. 6. (Color online) Influence of optical pulse intensity on the Auger electron spectra. The $\mathrm{x}$-ray pulse intensity is $I_{x}=$ $5 \times 10^{18} \mathrm{~W} / \mathrm{cm}^{2}$ for all cases, while the optical pulse intensity is $I_{\mathrm{opt}}=0($ dotted $), 0.5 \times 10^{12}$ (dashed), $0.8 \times 10^{12}$ (short-dashed), and $10^{12} \mathrm{~W} / \mathrm{cm}^{2}$ (solid), respectively.

into the continuum through process (C), and accordingly, the total electron yield significantly increases [dotted line in Fig. 5(a)] compared with the result with processes (A)-(C) all excluded [dot-dashed line in Fig. 5(a)]. If we include process (A) in addition to process (C) while process (B) is still excluded, the total electron yield increases as the optical pulse intensity increases [short-dashed line in Fig. 5(a)]. This is simply because the inclusion of process (A) opens a new channel which increases the electron yield through two-photon ionization from $|a\rangle$ by the optical pulse. Instead, if we include process (B) in addition to process (C) while excluding process (A), we notice the slight monotonic decrease of total electron yield as the optical pulse intensity increases [dashed line in Fig. 5(a)]. This is because the channel for population loss through process (A) is now closed, and accordingly CPT, although it is still imperfect, improves. We can infer from the above arguments, the processes (A) and (B) contribute to the total electron yield in an opposite manner, as the optical pulse intensity increases. This explains why the total electron yield for the full calculations, including all processes (A)-(C), remains almost constant as the optical pulse intensity is varied [solid line in Fig. 5(a)].

Having understood how the electron yield and the population dynamics are altered by the introduction of the optical pulse (Fig. 5), we now investigate the changes in the Auger electron spectra. For this purpose we vary the intensity of the optical pulse, while that of the $\mathrm{x}$-ray pulse is fixed at $I_{x}=5 \times 10^{18} \mathrm{~W} / \mathrm{cm}^{2}$. The results are shown in Fig. 6. Even for the case of the x-ray pulse alone (dotted line in Fig. 6), an asymmetric splitting already appears in the Auger electron spectrum, in which the asymmetry is a clear signature of the interference via two pathways, $|g\rangle \rightarrow|a\rangle \rightarrow\left|f_{1}\right\rangle$ and $|g\rangle \rightarrow\left|f_{1}\right\rangle$ (see Fig. 1), as reported in Ref. [11]. As the optical pulse intensity increases, the electron peaks reduce their heights and shift in opposite directions. Moreover, we notice that the line splitting at around $811.4 \mathrm{eV}$ is most prominent at the optical pulse intensity of $10^{12} \mathrm{~W} / \mathrm{cm}^{2}$, as 


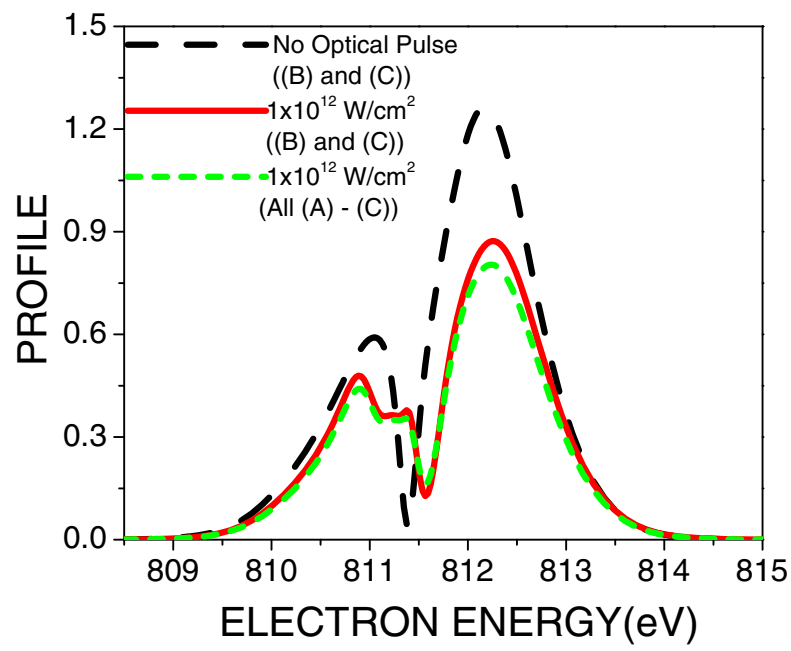

FIG. 7. (Color online) Influence of population loss from state $|a\rangle$ through photoionization by the optical pulse [process (A)] on the Auger electron spectra. The x-ray pulse intensity is $I_{x}=5 \times$ $10^{18} \mathrm{~W} / \mathrm{cm}^{2}$ for all cases. $I_{\mathrm{opt}}=0$ (dashed) with processes (B) and (C) only, $I_{\mathrm{opt}}=10^{12} \mathrm{~W} / \mathrm{cm}^{2}$ with processes (B) and (C) only (solid), and $I_{\mathrm{opt}}=10^{12} \mathrm{~W} / \mathrm{cm}^{2}$ with all processes $(\mathrm{A})-(\mathrm{C})$ (short-dashed), respectively.

we have mentioned before for Fig. 4. To clarify the origin of this line splitting, we carry out the calculations without and with the optical pulse. The results are shown in Fig. 7. Since no such line splitting is seen in the absence of the optical pulse (dashed line in Fig. 7), this small doubletlike structure at around $811.4 \mathrm{eV}$ can be identified as the ac Stark splitting induced by the optical pulse. This interpretation is further confirmed by the fact that the separation between the small peaks is nearly equal to the Rabi frequency by the optical pulse. Namely, the distortion around $811.4 \mathrm{eV}$ seen in Figs. 6 and 7 is a result of the superposition of the small ac Stark splitting induced by the optical pulse on the line splitting induced by the $\mathrm{x}$-ray pulse. From the comparison of the results without and with process (A) (solid and short-dashed lines in Fig. 7), we can say that the visibility of the distortion at around $811.4 \mathrm{eV}$ is slightly better if process (A) is excluded. In other words, the additional broadening of state $|a\rangle$ due to ionization by the optical pulse obscures the small structure at around $811.4 \mathrm{eV}$.

If the broadening of state $|a\rangle$ is the main cause of obscuring the subtle structure around $811.4 \mathrm{eV}$, ionization by the optical pulse [process (A)] is not the only mechanism for that. The short lifetime of state $|a\rangle, 2.4 \mathrm{fs}$, due to the fast Auger decay, would be another cause of not being able to see the clear splitting structure by the optical pulse. To test this argument, we hypothetically change the lifetime of state $|a\rangle$ and calculate the Auger electron spectra. The results are shown in Fig. 8. As we expected, the structure at around $811.4 \mathrm{eV}$ is less (more) visible if the lifetime of state $|a\rangle$ is set to be shorter (longer).

As we have described in Fig. 1, not only the interference via two pathways [due to the presence of process (C)] but also the additional photoionization processes from the coreexcited states [processes (A) and (B)] play important roles in the magnitude and shape of the Auger electron peaks. To clarify the contribution of the respective processes (A)-(C)

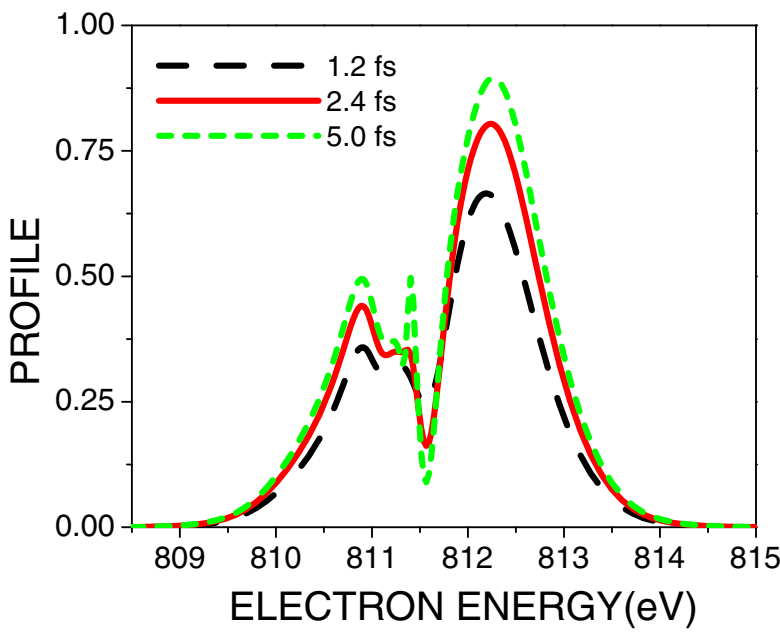

FIG. 8. (Color online) Influence of lifetime of state $|a\rangle$ on the Auger electron spectra. $I_{x}=5 \times 10^{18}$ and $I_{\mathrm{opt}}=10^{12} \mathrm{~W} / \mathrm{cm}^{2}$ for all cases. The lifetime of state $|a\rangle$ is hypothetically varied from the original value, $2.4 \mathrm{fs}$ (solid) to $1.2 \mathrm{fs}$ (dashed) and 5.0 fs (shortdashed), respectively.

to the Auger electron spectra, we include processes (A)-(C) step by step again and study the changes in the Auger electron spectra. The results are summarized in Fig. 9. If we include only processes (A) or (B) (short-dashed and dot-dashed lines in Fig. 9) we observe the double peaks with similar shapes but different heights. The difference in their heights mainly arises from the different populations in state $|a\rangle$ through ionization by the optical and $\mathrm{x}$-ray pulses. If we include both processes (A) and (B), but still without process (C), the Auger electron profile becomes less. This occurs due to reduction of population in state $|a\rangle$, through ionization by both x-ray and optical pulses, as a result of which a lesser fraction of the population in state

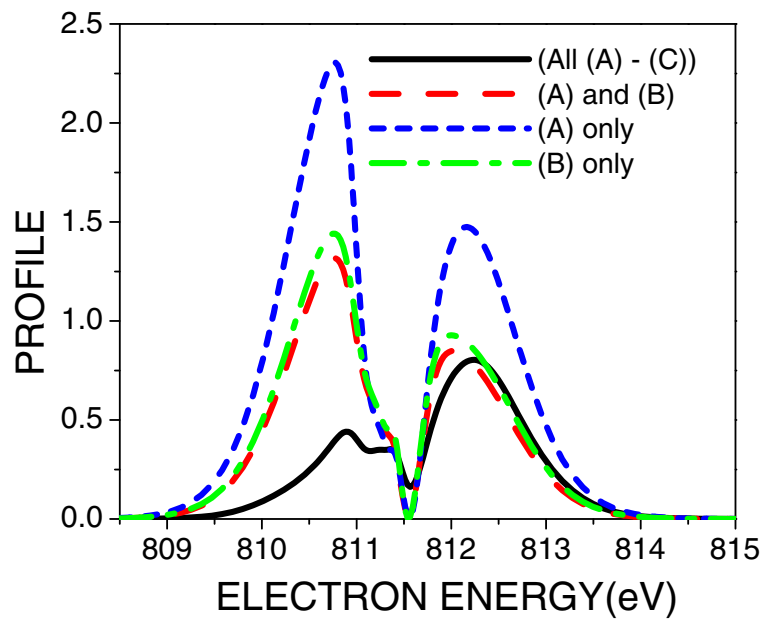

FIG. 9. (Color online) Modification of the Auger electron spectra due to the interference between two competing pathways, $|g\rangle \rightarrow$ $|a\rangle \rightarrow\left|f_{1}\right\rangle$ and $|g\rangle \rightarrow\left|f_{1}\right\rangle$ (in Fig. 1). $I_{x}=5 \times 10^{18} \mathrm{~W} / \mathrm{cm}^{2}$ and $I_{\mathrm{opt}}=10^{12} \mathrm{~W} / \mathrm{cm}^{2}$ for all cases. To highlight the interference effect, the included photoionization processes are all processes (A)-(C) (solid), processes (A) and (B) only (dashed), process (A) only (short-dashed), and process (B) only (dot-dashed), respectively. 
$|a\rangle$ experiences the RA decay. Interestingly, if we include the direct ionization process [process $(\mathrm{C})$ ] in addition to processes (A) and (B), the height of the Auger electron profile becomes even less. Not only that, the height of the left peak of the double peaks becomes smaller and the distortion in the shape of the left peak becomes more significant than that of the right one (solid line in Fig. 9). This is very different from the other three curves presented in Fig. 9. These results clearly show that the interference induced by the strong $\mathrm{x}$-ray pulse via the two competing pathways [11] is persistent but somehow modified through the coupling of the two core-excited states by the strong near-resonant optical pulse.

\section{CONCLUSIONS}

In conclusion, we have theoretically investigated the effects of strong couplings in resonant Auger processes under the strong bichromatic (x-ray + optical) field. There are two kinds of strong couplings involved in the processes. The one is the coupling between the ground and coreexcited states by the strong x-ray pulse, and the other is the coupling between the two core-excited states by the strong optical pulse. We have shown that the Auger electron spectrum changes its shape as the intensities of the X-ray and/or optical pulses increase, and at the sufficiently high intensities for both pulses the strong couplings manifest themselves in the emergence of the two splittings superimposed on the Auger electron spectra. However, if the x-ray pulse intensity is too high, the splitting associated with the optical pulse becomes more subtle due to the broadening of the core-excited state as a result of rapid photoionization (by both x-ray and optical pulse). We have also shown that the interference between the two competing pathways by the strong $\mathrm{x}$-ray pulse, which has been recently investigated for the case of the strong $\mathrm{x}$-ray pulse alone [11], is persistent but altered by the simultaneous presence of the strong optical pulse. Through the systematic study by including or excluding the different photoionization processes from the core-excited as well as ground states, we have also clarified the contribution of the respective processes to the total electron yield and the Auger electron spectra. Our results indicate that we are able to control the resonant Auger process through the introduction of the second core-excited state and the optical pulse.

\section{ACKNOWLEDGMENT}

This work was supported by a Grant-in-Aid for Scientific Research from the Ministry of Education, Culture, Sports, Science and Technology of Japan.
[1] G. B. Armen, H. Aksela, T. Åberg, and S. Aksela, J. Phys. B 33, R49 (2000).

[2] R. Camilloni, M. Žitnik, C. Comicioli, K. C. Prince, M. Zacchigna, C. Crotti, C. Ottaviani, C. Quaresima, P. Perfetti, and G. Stefani, Phys. Rev. Lett. 77, 2646 (1996).

[3] E. Kukk, H. Aksela, A. Kivimäki, J. Jauhiainen, E. Nõmmiste, and S. Aksela, Phys. Rev. A 56, 1481 (1997).

[4] N. Saito, N. M. Kabachnik, Y. Shimizu, H. Yoshida, H. Ohashi, Y. Tamenori, I. H. Suzuki, and K. Ueda, J. Phys. B 33, L729 (2000).

[5] B. M. Lagutin, Ph. V. Demekhin, V. L. Sukhorukov, A. Ehresmann, and H. Schmoranzer, J. Phys. B 36, L163 (2003).

[6] B. M. Lagutin, I. D. Petrov, V. L. Sukhorukov, S. Kammer, S. Mickat, R. Schill, K.-H. Schartner, A. Ehresmann, Yu. A. Shutov, and H. Schmoranzer, Phys. Rev. Lett. 90, 073001 (2003).

[7] B. M. Lagutin, I. D. Petrov, V. L. Sukhorukov, Ph. V. Demekhin, B. Zimmermann, S. Mickat, S. Kammer, K.-H. Schartner, A. Ehresmann, Yu. A. Shutov, and H. Schmoranzer, J. Phys. B 36, 3251 (2003).

[8] N. Rohringer and R. Santra, Phys. Rev. A 77, 053404 (2008).

[9] J.-C. Liu, Y.-P. Sun, C.-K. Wang, H. Ågren, and F. Gel'mukhanov, Phys. Rev. A 81, 043412 (2010).

[10] Y.-P. Sun, J.-C. Liu, C.-K. Wang, and F. Gel'mukhanov, Phys. Rev. A 81, 013812 (2010).

[11] Ph. V. Demekhin and L. S. Cederbaum, Phys. Rev. A 83, 023422 (2011).

[12] A. D. Müller and Ph. V. Demekhin, J. Phys. B 48, 075602 (2015).

[13] E. P. Kanter et al., Phys. Rev. Lett. 107, 233001 (2011).
[14] M. Drescher, M. Hentschel, R. Kienberger, M. Uiberacker, V. Yakovlev, A. Scrinzi, Th. Westerwalbesloh, U. Kleineberg, U. Heinzmann, and F. Krausz, Nature (London) 419, 803 (2002).

[15] M. Meyer et al., Phys. Rev. Lett. 108, 063007 (2012).

[16] T. Mazza, K. G. Papamihail, P. Radcliffe, W. B. Li, T. J. Kelly, J. T. Costello, S. Düsterer, P. Lambropoulos, and M. Meyer, J. Phys. B 45, 141001 (2012).

[17] A. Picón, C. Buth, G. Doumy, B. Krässig, L. Young, and S. H. Southworth, Phys. Rev. A 87, 013432 (2013).

[18] T. E. Glover, M. P. Hertlein, S. H. Southworth, T. K. Allison, J. van Tilborg, E. P. Kanter, B. Krässig, H. R. Varma, B. Rude, R. Santra, A. Belkacem, and L. Young, Nat. Phys. 6, 69 (2010).

[19] C. Buth, R. Santra, and L. Young, Phys. Rev. Lett. 98, 253001 (2007).

[20] J. M. Schins, P. Breger, P. Agostini, R. C. Constantinescu, H. G. Muller, G. Grillon, A. Antonetti, and A. Mysyrowicz, Phys. Rev. Lett. 73, 2180 (1994).

[21] O. Smirnova, V. S. Yakovlev, and A. Scrinzi, Phys. Rev. Lett. 91, 253001 (2003).

[22] A. K. Kazansky and N. M. Kabachnik, J. Phys. B 42, 121002 (2009).

[23] A. K. Kazansky and N. M. Kabachnik, J. Phys. B 43, 035601 (2010).

[24] C. Buth and K. J. Schafer, Phys. Rev. A 80, 033410 (2009).

[25] A. Picón, P. J. Ho, G. Doumy, and S. H. Southworth, New J. Phys. 15, 083057 (2013).

[26] V. F. Weisskopf and E. P. Wigner, Z. Phys. 63, 54 (1930).

[27] R. Santra and L. S. Cederbaum, Phys. Rep. 368, 1 (2002).

[28] Ph. V. Demekhin and L. S. Cederbaum, Phys. Rev. A 86, 063412 (2012). 